CLINICAL AND EXPERIMENTAL VACCINE RESEARCH

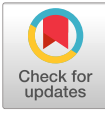

Clin Exp Vaccine Res 2019;8:132-135 https://doi.org/10.7774/cevr.2019.8.2.132 pISSN 2287-3651 • elSSN 2287-366X

Dong-Kun Yang', Ha-Hyun Kim', Eun-Jin Lee', Jae-Young Yoo', Jong-Taek Kim², Sangjin Ahn' ${ }^{1}$ Viral Disease Research Division, Animal and Plant Quarantine Agency, Ministry of Agriculture, Food and Rural Affairs, Gimcheon; ${ }^{2}$ College of Veterinary Medicine, Kangwon National University, Chuncheon, Korea

Received: March 5, 2019

Revised: July 4, 2019

Accepted: July 4, 2019

Corresponding author: Dong-Kun Yang, PhD, DVM Viral Disease Research Division, Animal and Plant Quarantine Agency, Ministry of Agriculture, Food and Rural Affairs, 177 Hyeoksin 8-ro, Gimcheon 39660 , Korea

Tel: +82-54-912-0785, Fax: +82-54-912-0812

E-mail: yangdk@korea.kr

No potential conflict of interest relevant to this article was reported.

This work was supported financially by a grant (N 1543085-2017-36-01) from the Animal, and Plant Quarantine Agency, Ministry of Agriculture, Food and Rural Affairs, Republic of Korea.

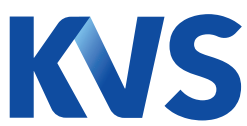

KOR E A N

VACCINE

SO CI E T Y

(c) Korean Vaccine Society.

This is an Open Access article distributed under the terms of the Creative Commons Attribution Non-Commercial License (http://creativecommons.org/licenses/ by-nc/4.0) which permits unrestricted non-commercial use, distribution, and reproduction in any medium, provided the original work is properly cited.

\section{Rabies immune status of raccoon dogs residing in areas where rabies bait vaccine has been distributed}

Since 2000, large amounts of rabies bait vaccine have been distributed in two provinces where raccoon dog-mediated rabies has occurred. A total of 146 raccoon dogs were caught in Gangwon and Gyeonggi Provinces from January 2017 to June 2018, and raccoon dog blood samples were collected. Of the 146 raccoon dogs, 13.7\% (20/146) had rabies antibodies. In Gyeonggi and Gangwon provinces, the rate of rabies antibody was $8.5 \%(5 / 59)$ and $17.2 \%$ (15/87), respectively. Considering these results, it would be desirable to improve the distribution method or use a new bait vaccine to prevent animal rabies in South Korea.

Keywords: Rabies, Immune status, Raccoon dogs

Among the diseases transmitted from animals to humans, rabies is the deadliest in both [1]. Rabies is caused by the rabies virus (RABV), belonging to the genus Lyssavirus in the family Rhabdoviridae [2]. Since the first animal rabies case was identified in Korea in 1907, a number of animal rabies cases had been reported in several species until 1984 [3]. No rabies cases were identified between 1985 and 1992. However, a dog that fought a raccoon dog (Nyctereutes procyonoides koreensis) in 1993 was diagnosed with rabies. Subsequently, the number of animal rabies cases in both domestic and wild animals increased around the de-militarized zone in Gangwon and Gyeonggi provinces, South Korea. After finding out that the raccoon dog was a host for rabies, the Korean government sought ways to reduce wildlife-mediated rabies [4]. In 2000, the government found that oral bait vaccines were effective in reducing rabies in wildlife and two oral rabies vaccines (ORVs) had been commercialized in Europe: SAG2 (Street Alabama Gif) and vaccinia RABV glycoprotein (VRG, Merial, Lyon, France) vaccines. After testing both ORVs in raccoon dogs, the VRG vaccine was selected for use in South Korea starting in 2000. The amount of VRG vaccine distributed has increased annually, and 99 million bait vaccines in 2018 had been spread. The number of rabies cases peaked at 78 cases in 2002 and decreased gradually until 2013. No rabies has occurred in South Korea since 2014 [4]. Despite that the rabies bait vaccine has contributed to the reduction in rabies cases, it has been required to investigate the immune status of raccoon dogs to prevent rabies re-emergence. In this study, we caught raccoon dogs living in Gyeonggi and Gangwon provinces, where the rabies bait vaccine has been distributed, and investigated the rabies immune status of the raccoon dogs.

To survey the RABV immune status of wild raccoon dogs residing in Gyeonggi and Gangwon provinces where the VRG has been distributed, animals were caught using 
catch traps (Boryeong, Korea), neck scoops, and control poles from January 2017 to June 2018. The body of captured raccoon dogs was measured and a microchip was inserted into the captured raccood dogs to identify individuals, and blood was collected from a cephalic or jugular vein. The coagulated blood was centrifuged at $3,000 \times \mathrm{g}$ for 10 minutes to separate serum, which was stored at $-20^{\circ} \mathrm{C}$ until used.

The virus-neutralizing antibody (VNA) titer against RABV was measured using a fluorescent antibody virus neutralization test [5]. Briefly, a dog reference serum sample positive for RABV was purchased from the French Agency for Food, Environmental and Occupational Health and Safety, and the VNA titer was adjusted to $0.5 \mathrm{IU} / \mathrm{mL}$ for use as a positive control. The sample from each raccoon dog was diluted in three fold with positive and negative serum samples. Then, standard RABV, the CVS-11 strain, was added to each microplate well at approximately $100 \mathrm{FAID}_{50} / 50 \mu \mathrm{L}$. After a 1-hour incubation at $37^{\circ} \mathrm{C}, 50 \mu \mathrm{L}$ of a suspension containing $4 \times 10^{5} \mathrm{BHK}-$ 21 cells/mL (ATCC CCL-10) were added to each well, and the plates were cultured under the same conditions for 72 hours. The plates were allowed to stand in cold acetone for $30 \mathrm{~min}$ utes to fix the BHK-21 cells and then reacted with a specific monoclonal antibody against RABV for 45 minutes at $37^{\circ} \mathrm{C}$ after rinsing three times with phosphate-buffered saline (PBS; $\mathrm{pH}$ 7.2). Then, the plates were stained with FITC-conjugated goat anti-mouse IgG+IgM. After washing with PBS, the specific fluorescence in the BHK-21 cells infected with RABV was observed at $400 \times$ under a fluorescence microscope (Nikon, Tokyo, Japan). After comparing the results with those of the RABV-positive reference standard, the VNA titers (IU/mL) were determined. The chi-square test was used to analyze differences in seroprevalence stratified by geographic region. Statistical significance was defined by $\mathrm{p}<0.05$.

In total, 146 raccoon dogs were caught in 13 counties in Gyeonggi (59) and 14 counties in Gangwon (87). Gangneung (34) and Suwon (13) were the counties where the most rac-

Table 1. Mean body weight, height, and length of the Korean raccoon dogs caught in the regions at risk for rabies

\begin{tabular}{lccc}
\hline Province & Body weight $(\mathrm{kg})$ & Height $\left(\mathrm{cm}^{2}\right)$ & Length $\left(\mathrm{cm}^{2}\right)$ \\
\hline Gyeonggi (31) & $3.45 \pm 0.85$ & $20.27 \pm 3.49$ & $29.42 \pm 4.33$ \\
Gangwon (80) & $3.87 \pm 0.97$ & $21.53 \pm 3.25$ & $32.17 \pm 5.43$ \\
Total (111) & $3.75 \pm 0.96$ & $21.18 \pm 3.33$ & $31.40 \pm 5.28$
\end{tabular}

alThe value in parentheses is the number of raccoon dogs captured. The difference (35) between the number of serum samples and number of body measurements is due to the poor accuracy of the physical measurements performed after blood collection. coon dogs were captured in Gyeonggi and Gangwon, respectively. The body weight, height, and length of 111 raccoon dogs were measured, with averages of $3.75 \mathrm{~kg}, 21.2 \mathrm{~cm}$, and $31.4 \mathrm{~cm}$, respectively (Table 1). The larger the body size and the older the raccoon dog, the more likely it is to take the bait vaccine. This is becasuse the VRG block ( $33 \mathrm{~mm} \times 33 \mathrm{~mm} \times 20$ $\mathrm{mm}$ ) consistng of fishmeal bait, wax and plasitc sachets is unfamiliar to young raccoon dogs that are difficult to swaloow [6]. The raccoon dogs caught in Gangwon were on average heavier, taller, and longer than those caught in Gyeonggi. Table 2 shows the RABV seropositive rates. The average seropositive rate for RABV was $13.7 \%$ overall. The regional seropositivity for raccoon dogs was $25.0 \%$ (1/4) in Gwangju, $15.4 \%$

Table 2. Regional distribution of RABV seropositivity in raccoon dogs caught in Gyeonggi and Gangwon provinces

\begin{tabular}{|c|c|c|}
\hline & VNA titer (IU/mL) & No. of positive/tested (\%) \\
\hline \multicolumn{3}{|l|}{ County in Gyeonggi } \\
\hline Gapyeong & - & $0 / 4(0.0)$ \\
\hline Gwangmyeong & 0.87 & $1 / 7(14.3)$ \\
\hline Gwangju & 0.5 & $1 / 4(25.0)$ \\
\hline Gunpo & - & $0 / 1(0.0)$ \\
\hline Seongnam & - & $0 / 2(0.0)$ \\
\hline Suwon & $0.5,0.5$ & $2 / 13(15.4)$ \\
\hline Ansan & - & $0 / 1(0.0)$ \\
\hline Osan & - & $0 / 1(0.0)$ \\
\hline Yongin & 0.5 & $1 / 11(9.1)$ \\
\hline Pyeongtaek & - & $0 / 7(0.0)$ \\
\hline Pocheon & - & $0 / 3(0.0)$ \\
\hline Hwaseong & - & $0 / 4(0.0)$ \\
\hline Incheon & - & $0 / 1(0.0)$ \\
\hline Total & 0.57 & $5 / 59(8.5)$ \\
\hline \multicolumn{3}{|c|}{ County in Gangwon } \\
\hline Gangneung & $0.5,1.5,7.9,1.5,0.87,1.5$ & 6/34 (17.6) \\
\hline Donghae & - & $0 / 3(0.0)$ \\
\hline Sokcho & $0.5,1.5$ & $2 / 2(100)$ \\
\hline Yanggu & - & $0 / 2(0.0)$ \\
\hline Yangyang & - & $0 / 1(0.0)$ \\
\hline Yeongwol & - & $0 / 1(0.0)$ \\
\hline Wonju & - & $0 / 2(0.0)$ \\
\hline Jeongseon & - & $0 / 1(0.0)$ \\
\hline Cheorwon & $23.9,0.5$ & 2/7 (28.6) \\
\hline Chuncheon & $4.6,0.5,41.6$ & $3 / 20(15.0)$ \\
\hline Pyeongchang & - & $0 / 0(0.0)$ \\
\hline Hongcheon & 1.5 & $1 / 5(20.0)$ \\
\hline Hwacheon & 125 & 1/5 (20.0) \\
\hline Hoengseong & - & $0 / 2(0.0)$ \\
\hline Total & 14.2 & 15/87 (17.2) \\
\hline
\end{tabular}

RABV, rabies virus; VNA, virus-neutralizing antibody. 
(2/13) in Suwon, 14.3\% (1/7) in Gwangmyeong, and 9.1\% (1/11) in Yongin County, Gyeonggi Province, and $100 \%(2 / 2)$ in Sokcho, 28.6\% (2/7) in Cheorwon, 20.0\% (1/5) each in Hongcheon and Hwacheon, 17.6\% (6/34) in Gangneung, and 15.0\% (3/20) in Chuncheon County, Gangwon Province. No seropositive animals were found in Gapyeong, Gunpo, Seongnam, Ansan, Osan, Incheon, Pyeongtaek, or Hwaseong counties in Gyeonggi Province or in Donghae, Yanggu, Yangyang, Yeongwol, Wonju, Jeongseon, Pyeongchang, or Hoengseong counties in Gangwon Province. More raccoon dogs were seropositive in Gangwon Province (17.2\%; 15/87) compared with Gyeonggi Province $(8.5 \% ; 5 / 59)$. Among the raccoon dogs captured in Gyeonggi and Gangwon provinces, the mean VNA titers of the raccoon dogs seropositive for RABV were 0.57 and 14.2 $\mathrm{IU} / \mathrm{mL}$, respectively.

ORVs have been used to prevent wildlife-mediated rabies. As of 2019, there are four commercial ORVs for raccoons, foxes, and raccoon dogs: SAG2 (Street Alabama Gif), recombinat vacciniavirus (VRG), recombinant adenovirus (ONRAB) and recombinant rabies virus (SPBN GASGAS: Rabitec) in the world. The ONRAB (Artemis Technologies, Guelph, ON, Canada) was licensed for use with free-ranging striped skunks in Canada and efficacy of the ONRAB was demonstrated in the raccoon. The Rabitec containing a recombinant rabies virus engineered with a reverse genetic system was registered as a bait vaccine for red fox and raccoon dog in Germany in 2017 [7]. The VRG vaccine was developed in the 1980s and has been used in Belgium, France, Luxembourg, Israel, and North America for coyotes, foxes, and raccoons [8]. In France, the VRG bait vaccine was used from 1990 to 2005, and rabies has not occurred there since 1996. Belgium and Luxembourg also began distributing VRG vaccine by air in the early 1990s and continued until 2002 or 2003. These two countries were declared free of fox rabies in 2001 [9]. In the United States, VRG was approved as a rabies bait vaccine for raccoons in 1997 and is continuously distributed once a year (AugustSeptember) [10]. Like the countries mentioned above, South Korea has been distributing VRG vaccines to two rabies-risk provinces since 2000 . This policy suggests that ORVs led to the non-occurrence of wildlife rabies. Despite no animal rabies occurring in Korea, a serological survey to investigate the rabies immune status of raccoon dogs in the two provinces where the VRG vaccine has been distributed is an important tool for evaluating the success of the ORV program.

In this study, we measured the rabies VNA titers in raccoon dogs caught in areas where the rabies bait vaccine has been distributed. Of the 146 raccoon dogs examined, $13.7 \%$ (20/146) expressed rabies antibodies as a result of distributing the VRG bait. The seropositivity of Korean raccoon dogs in this study was lower than the rate (40\%) reported in 2012 [11] and that (38\%) in the United States in 2013 [12]. There may be several reasons for the low seropositivity in raccoon dogs. First, the level of rabies antibodies peaks 4-6 weeks after oral vaccination and then begins to decline [13]. The samples collected 6

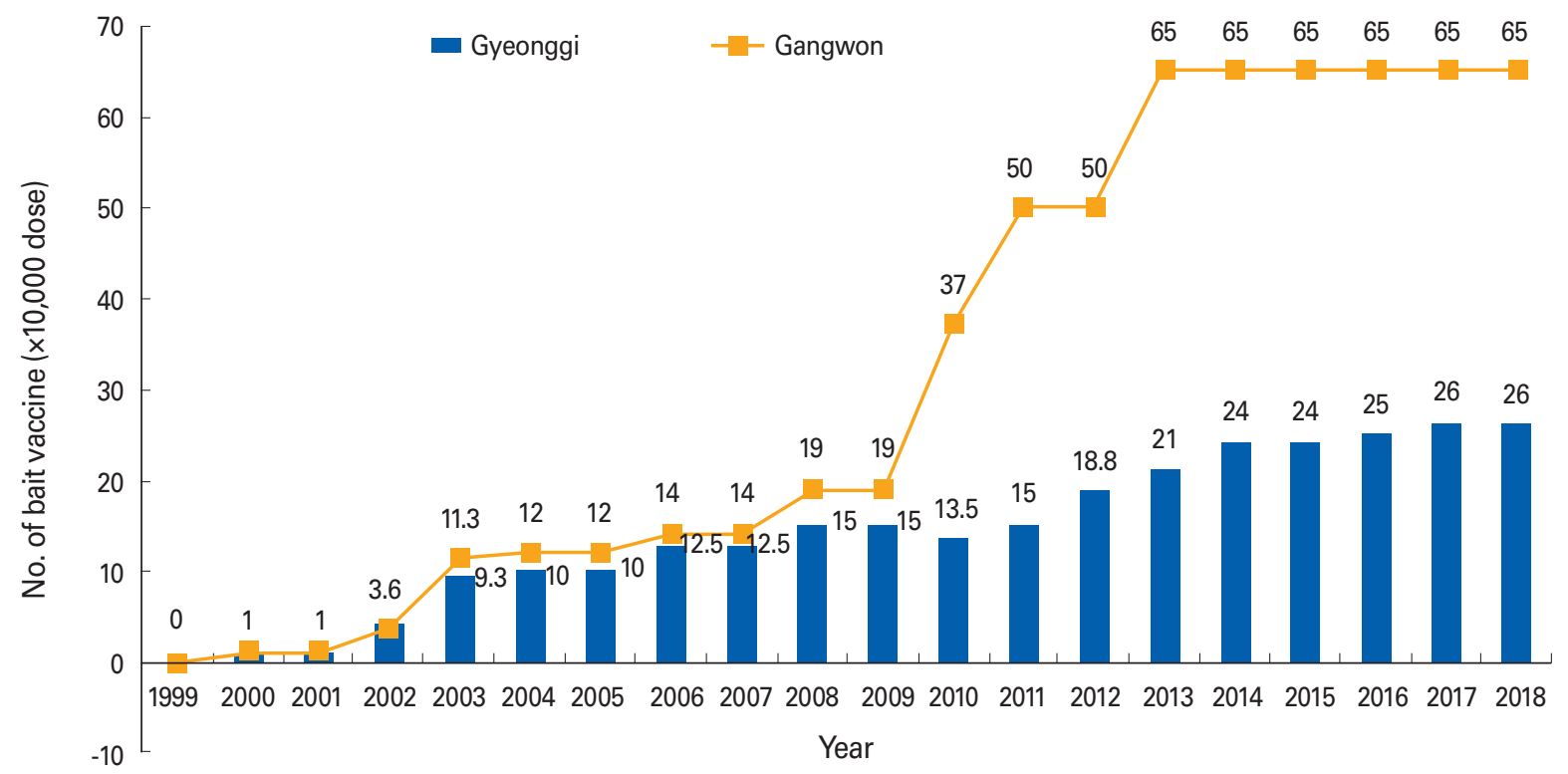

Fig. 1. Amount of rabies bait vaccine distributed since 2000 in Gyeonggi and Gangwon provinces. Data on the annual dose of bait vaccine were collected from the Planning and Implementation of Livestock Prevention Project issued by the Korean government. 
weeks after ingesting the bait vaccine may reflect the rabies immune status. Second, there are several ways to deliver the bait to target animals. Most countries that have adopted ORV programs use airplanes and helicopters [6]. However, the VRG vaccine has been distributed by hand placement around farms by people with local geographical knowledge. Consequently, discrepancies between the distribution area and capture site may affect the serological survey. In addition, fewer raccoon dogs were seropositive in Gyeonggi Province (8.5\%) than in Gangwon (17.2\%), indicating that the amount of bait distributed annually can affect the seroprevalence rate. As shown in Fig. 1, 2.5 times more bait was distributed in Gangwon than in Gyeonggi.

In conclusion, rabies can be prevented if animals have a blood VNA titer $\geq 0.5 \mathrm{IU} / \mathrm{mL}$ [14]. Only $13.7 \%$ of the raccoon dogs caught in the two provinces in this study had protective VNA titers, and this seropositivity was significantly lower than the $40 \%$ surveyed in the two mentioned provinces in 2012 [11], suggesting that the current ORV program should be improved to enhance immunity against rabies in Korean raccoon dogs. Application of a new bait vaccine might be required, as Canada replaced the VRG vaccine with ONRAB based on a comparison of bait vaccine performance and the United States has also conducted field trials of the ONRAB vaccine from 2012 to 2014 [15]. Second, it is necessary to implement regulations related to re-establishing regions at risk for rabies and a buffer zone near the de-militarized zone. A national rabies control program using an improved ORV may lead to the declaration that South Korea is rabies free.

\section{ORCID}

Dong-Kun Yang https://orcid.org/0000-0001-5765-3043

Ha-Hyun Kim https://orcid.org/0000-0001-6473-0035

Eun-Jin Lee https://orcid.org/0000-0002-5003-5389

Jae-Young Yoo https://orcid.org/0000-0001-7119-5421

Jong-Taek Kim https://orcid.org/0000-0002-6388-550X

Sangjin Ahn https://orcid.org/0000-0003-2398-2249

\section{References}

1. Fooks AR, Banyard AC, Horton DL, Johnson N, McElhinney LM, Jackson AC. Current status of rabies and prospects for elimination. Lancet 2014;384:1389-99.

2. Bourhy H, Kissi B, Tordo N. Taxonomy and evolutionary studies on lyssaviruses with special reference to Africa.
Onderstepoort J Vet Res 1993;60:277-82.

3. Kim CH, Lee CG, Yoon HC, et al. Rabies, an emerging disease in Korea. J Vet Med B Infect Dis Vet Public Health 2006; 53:111-5.

4. Yang DK, Kim HH, Cho IS. Strategies to maintain Korea's animal rabies non-occurrence status. Clin Exp Vaccine Res 2018;7:87-92.

5. Office International des Epizooties. Manual of diagnostic tests and vaccines for terrestrial animals (rabies). 7th ed. Paris: Office International des Epizooties; 2012. p.263-82.

6. Maki J, Guiot AL, Aubert M, et al. Oral vaccination of wildlife using a vaccinia-rabies-glycoprotein recombinant virus vaccine (RABORAL V-RG((R))): a global review. Vet Res 2017;48:57.

7. Ortmann S, Kretzschmar A, Kaiser C, et al. In vivo safety studies with SPBN GASGAS in the frame of oral vaccination of foxes and raccoon dogs against rabies. Front Vet Sci 2018;5:91.

8. Rupprecht CE, Hanlon CA, Slate D. Oral vaccination of wildlife against rabies: opportunities and challenges in prevention and control. Dev Biol (Basel) 2004;119:173-84.

9. Cliquet F, Aubert M. Elimination of terrestrial rabies in Western European countries. Dev Biol (Basel) 2004;119:185-204.

10. Sterner RT, Meltzer MI, Shwiff SA, Slate D. Tactics and economics of wildlife oral rabies vaccination, Canada and the United States. Emerg Infect Dis 2009;15:1176-84.

11. Oh SY, Kim SA, Kim JY, Yoo HS, Lee KK, Shin NS. Detection of antibodies against the rabies virus in Korean raccoon dogs (Nyctereutes procyonoides koreensis). J Zoo Wildl Med 2012;43:174-6.

12. Mainguy J, Fehlner-Gardiner C, Slate D, Rudd RJ. Oral rabies vaccination in raccoons: comparison of ONRAB(R) and RABORAL V-RG(R) vaccine-bait field performance in Quebec, Canada and Vermont, USA. J Wildl Dis 2013;49: 190-3.

13. Hsu AP, Tseng CH, Barrat J, et al. Safety, efficacy and immunogenicity evaluation of the SAG2 oral rabies vaccine in Formosan ferret badgers. PLoS One 2017;12:e0184831.

14. Lalosevic D, Lalosevic V, Lazarevic-Ivanc L, Knezevic I. BHK-21 cell culture rabies vaccine: immunogenicity of a candidate vaccine for humans. Dev Biol (Basel) 2008;131: 421-9.

15. Gilbert AT, Johnson SR, Nelson KM, et al. Field trials of Ontario rabies vaccine bait in the Northeastern USA, 201214. J Wildl Dis 2018;54:790-801. 